\title{
Abdominal wall sarcoma: A rare tumor and surgical treatment proposed
}

\author{
Roriz-Silva $\mathrm{R}^{1 *}$, Furuno da Silva $\mathrm{H}^{2}$ and Ramagem $\mathrm{CA}^{3}$ \\ ${ }^{1}$ Professor of General And Digestive Surgery, Federal University of Rondonia, Division of General Surgery, Hospital de Base, Porto Velho City, Brazil \\ ${ }^{2}$ General Surgeon, Division Of General Surgery, Hospital João Paulo II , Porto Velho City, Brazil \\ ${ }^{3}$ Professor of Oncology Surgery, Federal University of Rondonia, Hospital do Cancer de Barretos PVH, Brazil
}

\begin{abstract}
Abdominal wall sarcoma is part of a heterogeneous group of rare malignant neoplastic conditions with differentiated morphological patterns and originating from mesenchymal tissues. Soft tissue sarcomas are more prevalent in the lower and upper limbs and retroperitoneum but rarely occurring on the abdominal wall. This case corresponds to a 46-year-old patient, without associated comorbidities, complaining of painless swelling in the right flank with onset occurring 2 months previously. The computed tomography (CT) scan revealed a solid tumor on the abdominal wall of the flank measuring $10 \mathrm{x} 12 \mathrm{~cm}$, and the biopsy of the mass identified: highgrade myxoid sarcoma. The patient underwent surgical resection of most of the lateral abdominal wall with closure of the defect using polypropylene mesh, evolving satisfactorily in the postoperative period, and remains under follow-up for three years without local recurrence or metastases.
\end{abstract}

\section{Introduction}

Sarcomas are a heterogeneous group of rare and malignant neoplastic conditions with greater prevalence in adult male individuals [1]. Soft tissue sarcomas correspond to $1 \%$ of malignant tumors in adults [2], and occurrence on the abdominal wall is considered rare as they correspond to $0.1 \%$ of malignant tumors [1]. This group of neoplasms has its origin in mesenchymal cells of various sites: adipocytes, muscles, blood vessels, cartilage, nerves and bones [1]. The potential for destruction and recurrence of the tumor depends on the histological type and degree of mitoses, and immunohistochemical analysis is important particularly to define the source of the tumor and prognostic for the patients [3].

This report contains a description of a case of abdominal wall sarcoma and the proposed treatment with presentation of a large mass on the right flank.

\section{Case summary}

Patient EBS, male gender, age 46, of mixed race, without previous comorbidities, was referred by the public basic healthcare network to the surgical service of the Hospital de Base of Porto Velho, Rondônia state, Brazil. The patient complained of a painless large palpable nodule in the right flank and having first appeared 2 months previously, without other associated symptoms. The patient was a nonsmoker and did not have a family history of neoplasms. The abdominal computed tomography (CT) scan revealed a solid tumor of the abdominal wall involving the anterolateral muscles, measuring about $10 \mathrm{~cm} \times 12 \mathrm{~cm}$ in its major diameters, suggestive of primary abdominal wall tumor (Figure 1). The chest CT scan showed no evidence of pulmonary metastases. The needle biopsy of the center of the tumor revealed a high-grade myxoid sarcomatous tumor.

The patient underwent monobloc resection with elliptical incision of all the layers of the abdominal wall including the skin and the peritoneum of the anterolateral region of the abdomen, extending to the right lumbar region and segment of the $12^{\text {th }}$ rib and of a small portion of diaphragm insertion in the region of the resected muscles (Figure 2). The abdominal wall reconstruction was performed using 30 $\mathrm{x} 30 \mathrm{~cm}$ polypropylene mesh after suturing the greater omentum along the entire edge of the abdominal wall defect in order to prevent the intestinal loops from adhering to the prosthesis. The mesh was fixed in place with 2.0 nonabsorbable polypropylene sutures at separate points (Figure 3). Primary closure of the skin was performed using 4.0 nylon sutures, and it was necessary to perform water seal pleural drainage due to opening of the right pleural cavity during the procedure. The patient progressed satisfactorily, was discharged on the $4^{\text {th }}$ postoperative day following removal of the chest drain, and was followed up in the postoperative period for three years without local recurrence or distant metastases (Figure 4).

\section{Discussion}

Abdominal wall sarcomas manifest as a painless abdominal mass of progressive growth, and are generally diagnosed when they reach a size of more than $5 \mathrm{~cm}$ when they are easily palpable. The main differential diagnosis of these sarcomas are abdominal wall hernias besides benign tumors, mainly the so-called fibromatoses (Desmoid tumor) [1]. The presence of a painless mass on the abdominal wall with a size above 5 $\mathrm{cm}$ increases the chance of malignancy [4], and this fact was also been observed in the reported case, where there was a large painless mass in the right flank.

Correspondence to: Professor Roriz-Silva R, MSc, MD, Professor of general and Digestive Surgery, Federal University of Rondonia, Division of General Surgery, Hospital de Base, Porto Velho City, Brazil, Tel/Fax: 55 (69) 32165446, E-mail: roriz-silva@unir.br

Key words: abdominal wall sarcoma, soft tissue sarcoma, surgical approach

Received: July 22, 2017; Accepted: August 17, 2017; Published: August 19, 2017 


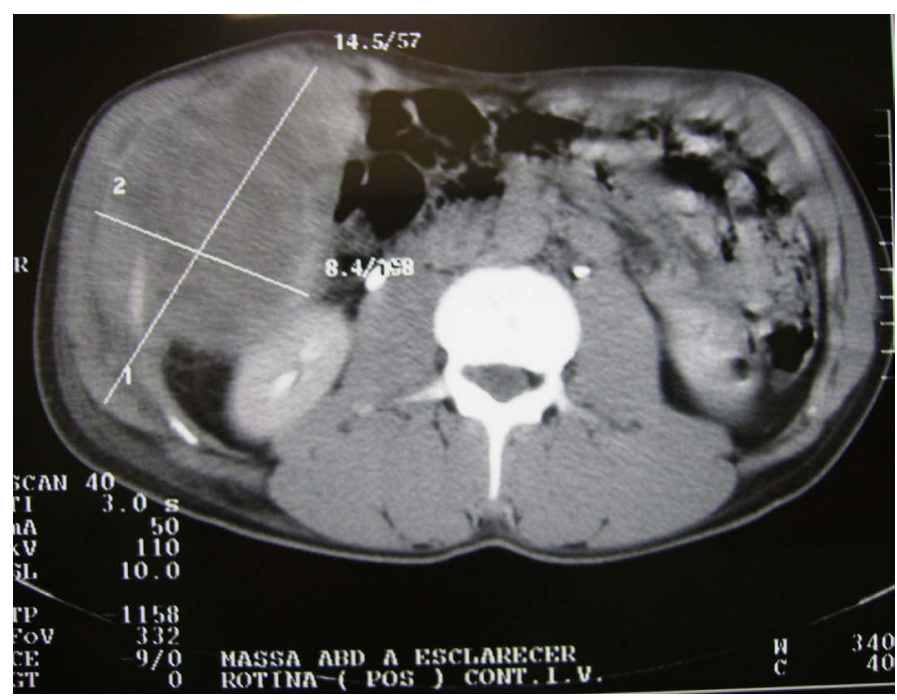

Figure 1. Computed tomography scan of abdomen showing tumor with heterogeneous density on anterolateral abdominal wall (right flank), measuring $12 \times 10 \mathrm{~cm}$.

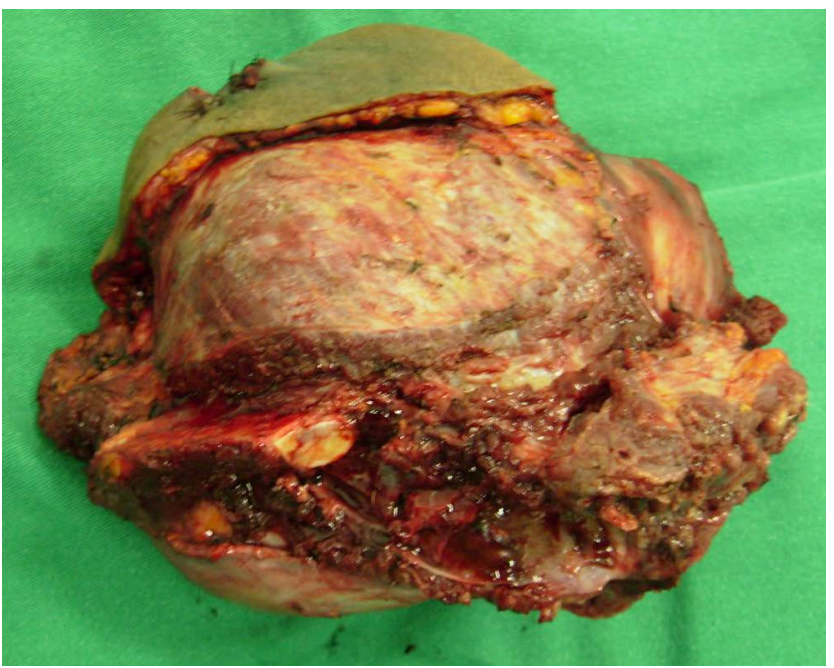

Figure 2. Surgical specimen with all the layers of the abdominal wall in the flank together with the segment of the $12^{\text {th }}$ resected right rib (arrow).

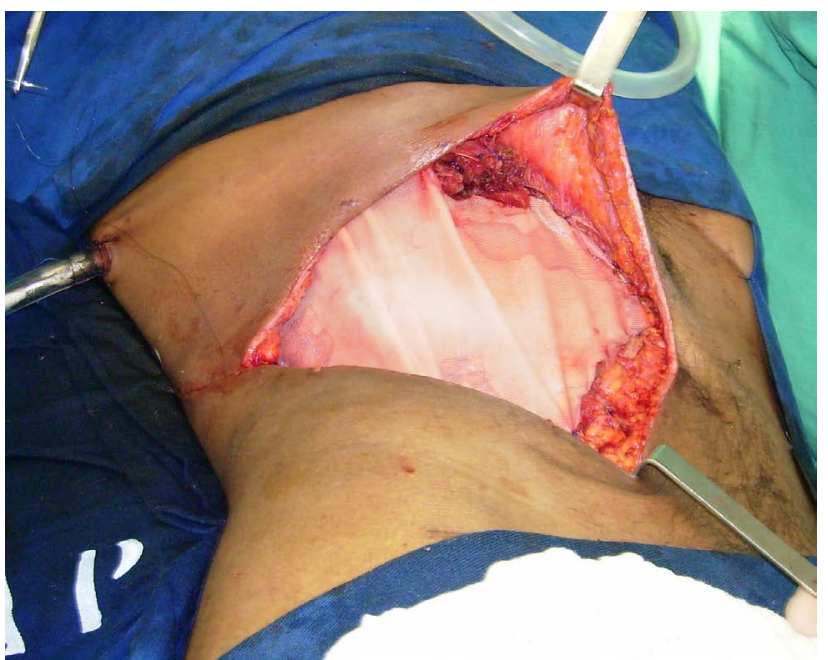

Figure 3. Reconstruction of the wall with fixing of the polypropylene mesh anterior to aponeurosis and suturing of the greater omentum inside the wall defect. Presence of pleural drainage associated with the procedure.

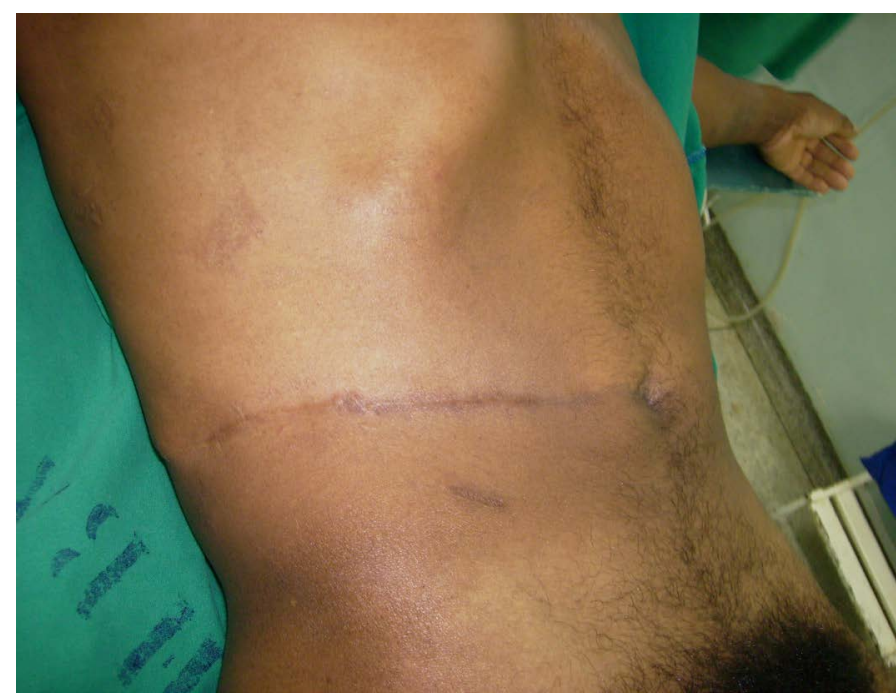

Figure 4. Outcome after three years of the surgical procedure.

In the diagnostic evaluation of sarcomas of extremities, nuclear magnetic resonance (NMR) imaging has specificity superior to CT scans, yet in abdominal wall tumors, the abdominal CT scan allows a better evaluation of the tumor's relationship with vital organs and intraabdominal structures such as the intestines [1]. All confirmed cases should also undergo a chest CT scan for pulmonary metastases [5].

The biopsy of the suspicious abdominal mass should be performed prior to surgical resection in all cases using and has a sensitivity of 80 to $95 \%$, in the definition of the presence or absence of malignancy [3]. The most suitable method is core needle biopsy of the tumor using one or more punches. Tumor subtype was accurately assigned in $89.5 \%$ of benign tumors and $88.0 \%$ of sarcomas [6]. That yet the puncture site should be included in the piece to be resected [1]. In the case reported here, the diagnostic method of choice was core needle biopsy of the tumor alongside a histopathological and immunohistochemical study.

The treatment of abdominal wall sarcoma consists of the en bloc resection of the lesion with a minimum margin of $3 \mathrm{~cm}$ of free tissue, involving the entire width of the abdominal wall, and possibly also involving the resection of adjacent structures or even internal organs, if there is tumor adherence or invasion [1,7]. Abdominal wall reconstruction can be performed using myocutaneous flaps or synthetic mesh, or a combination of both methods $[1,4]$. Nowadays the use of double-sided abdominal mesh is indicated most often in the closure of major abdominal wall defects with the purpose of avoiding adherences of intestinal loops to the screen and fistula formation. Simple polypropylene mesh (only one available in the service at the time) was used in the case in question, but the use of the greater omentum with suturing all along the edge of the abdominal wall defect and the anterior positioning of the screen was a simple yet effective surgical tactic to prevent the mesh from adhering to intestinal loops [1].

Tumors classified by histology as high grade and with a diameter greater than $5 \mathrm{~cm}$ are related to the development of metastasis, particularly of the pulmonary kind [1]. Another aspect that hinders the curing of sarcomas is the risk of local recurrence due to compromised surgical margins, even if these are microscopic called R1 resection [7]. Otherwise R0 resection is ideal goal and it can be enhancer 5-year rates of free of tumor for more then $90 \%$ [7]. The patient studied in this case exhibited free surgical margins and has been followed up now for three years without local recurrence or distant metastasis. 


\section{Conclusion}

Sarcoma of abdominal wall is a rare presentation of soft issue tumors and needs aggressive resection to prevent local recurrence. Reconstruction of abdominal wall requires adequate surgical approach and a long follow up of patients in order to identify local recurrence or metastasis.

\section{Conflicts of interest}

None declared.

\section{References}

1. Williams KJ, Hayes AJ (2014) A guide to oncological management of soft tissue tumours of the abdominal wall. Hernia 18: 91-97. [Crossref]

2. Wilmar JS, Manoel, Silveira Júnior BJQ, Abreu LP, Abreu Neto DCB, et al. (2008) Sarcomas de alto grau: estudo retrospectivo de 131 casos. Rev Col Bras Cir 35: 83-87.
3. Salas SB, Stoeckle B, Terrier E, Collin P, Leroux F, at al. (2009) Soft tissue sarcomas of the trunk wall (STS-TW): a study of 343 patients from the French Sarcoma Group (FSG) database. Ann Oncol 20: 1127-1135. [Crossref]

4. Stojadinovic A, Hoos A, Karpoff HM, Leung DH, Antonescu CR, et al. (2001) Soft tissue tumors of the abdominal wall: analysis of disease patterns and treatment. Arch Surg 136: 70-79. [Crossref]

5. García Del Muro X, Martín J, Maurel J, Cubedo R, Bagué S, et al. (2011) Soft tissue sarcomas: clinical practice guidelines. Med Clin (Barc) 136: 408. [Crossref]

6. Strauss DC, Qureshi YA, Hayes AJ, Thway K, Fisher C, et al. (2010) The role of core needle biopsy in the diagnosis of suspected soft tissue tumours. J Surg Oncol 102: 523-529. [Crossref]

7. O’Donnell PW, Griffin AM, Eward WC, Sternheim A, Catton, CN, Chung PW (2014) The effect of the setting of a positive surgical margin in soft tissue sarcoma. Cancer 120: 2866-2875. [Crossref]

Copyright: $@ 2017$ Roriz-Silva R. This is an open-access article distributed under the terms of the Creative Commons Attribution License, which permits unrestricted use, distribution, and reproduction in any medium, provided the original author and source are credited. 